\title{
Investigation of Acoustical Shielding by a Wedge-Shaped Airframe
}

by

\author{
Carl H. Gerhold* \\ Lorenzo R. Clark $^{* *}$ \\ NASA Langley Research Center \\ Hampton, Virginia
}

\author{
Mark H. Dunn ${ }^{* * *}$ \\ John Tweed T*** $^{* * 2}$ \\ Old Dominion University \\ Norfolk, Virginia
}

\begin{abstract}
Experiments on a scale model of an advanced unconventional subsonic transport concept, the Blended Wing Body (BWB), have demonstrated significant shielding of inlet-radiated noise. A computational model of the shielding mechanism has been developed using a combination of boundary integral equation method (BIEM) and equivalent source method (ESM). The computation models the incident sound from a point source in a nacelle and determines the scattered sound field. In this way the sound fields with and without the airfoil can be estimated for comparison to experiment. An experimental test bed using a simplified wedge-shape airfoil and a broadband point noise source in a simulated nacelle has been developed for the purposes of verifying the analytical model and also to study the effect of engine nacelle placement on shielding. The experimental study is conducted in the Anechoic Noise Research Facility at NASA Langley Research Center. The analytic and experimental results are compared at 6300 and 8000 $\mathrm{Hz}$. These frequencies correspond to approximately $150 \mathrm{~Hz}$ on the full scale aircraft. Comparison between the experimental and analytic results is quite good, not only for the noise scattering by the airframe, but also for the total sound pressure in the far field. Many of the details of the sound field that the analytic model predicts are seen or indicated in the experiment, within the spatial resolution limitations of the experiment. Changing nacelle location produces comparable changes in noise shielding contours evaluated analytically and experimentally. Future work in the project will be enhancement of the analytic model to extend the analysis to higher frequencies corresponding to the blade passage frequency of the high bypass ratio ducted fan engines that are expected to power the BWB.

\footnotetext{
${ }_{* * *}^{*}$ Senior Research Engineer, Senior member, AIAA, ${ }_{* * * * *}$ Aerospace Engineer

${ }^{* * *}$ Research Professor, Member, AIAA, ${ }^{* * * *}$ Professor

Copyright 2004 by the American Institute of Aeronautics and Astronautics, Inc. No copyright is asserted in the United States under Title 17, U.S. Code. The U.S. Government has a royalty-free license to exercise all rights under the copyright claimed herein for government purposes. All other rights are reserved by the copyright owner.
} 


$\begin{array}{ll}\text { Symbols } & \\ B & \\ B & \text { Point set describing the scattering surface } \\ B_{e} & \text { Point set describing the scattering body exterior } \\ B_{i} & \text { Point set describing the scattering body interior } \\ c & \text { Ambient sound speed } \\ k & \text { Source excitation wavenumber } \\ P & \text { Acoustic pressure amplitude } \\ P_{i n c} & \text { Incident acoustic pressure amplitude } \\ P_{s} & \text { Scattered acoustic pressure amplitude } \\ \vec{P}_{s} & \text { Approximate scattered acoustic pressure amplitude } \\ \left\{\vec{x}_{j}\right\}_{j=1}^{M} & \text { Source locations in scattering body interior } \\ \left\{\vec{y}_{j}\right\}_{j=1}^{N} & \text { Collocation points on scattering surface } \\ \square & \text { Boundary error function } \\ \square & \text { Source excitation frequency }\end{array}$

\section{Introduction}

Increasing awareness of aircraft noise in areas surrounding airports has led to renewed emphasis on aircraft noise reduction at the source. In order to meet NASA's aggressive noise impact reduction goals of the next 20 years, noise reduction must be an integral part of the aircraft design process. The Blended Wing Body is an unconventional transport concept that has the potential to address long-term NASA goals for emissions, safety, capacity, cost of travel, and noise. The Blended Wing Body combines a rigid, wide airfoil shape fuselage with high aspect ratio wings and semi-buried engines. A typical installation calls for high bypass-ratio engines mounted on top of the wing, aft of the passenger compartment. Inlet radiated noise is shielded in the area below the aircraft by the wing upper surface.

A previous study by Clark and Gerhold (1999) on an early model of the Blended Wing Body concept airframe verified significant shielding of the inlet-radiated noise by the fuselage. In addition to verifying inlet noise shielding, the data were to be used to validate an analytical model (Dunn, et. al., 1999). Initial comparison of measured to estimated noise shielding was encouraging. However, some discrepancies were noted and it was not clear whether they arose due to deficiencies in the analytic model or from the highly complex contour of the BWB model. Thus a simplified model of the BWB was developed and tested. The model serves two purposes, one of which is to provide sound scattering data to validate the analytic model. The other purpose of the simplified physical model is to provide a database of the effect of installation on engine noise radiated from aircraft during flyover. This paper discusses the analytical development and comparison between the theoretical and experimental results for the wedgeshaped airfoil noise shielding. 


\section{Experimental Model}

\section{a. Description of the Model}

Figure 1 shows a photograph of the model in the anechoic chamber of the Anechoic Noise Research Facility (ANRF) at NASA Langley Research Center. The circular hoop in the foreground is a microphone array that will be discussed in the next section. The wedge-shape airfoil is a triangular planform of length $36 \mathrm{in} .(914.4 \mathrm{~mm})$ and $72 \mathrm{in}$. (1828.8 mm) wingspan. It is 8 in. $(203.2 \mathrm{~mm})$ high at the center of gravity and tapers to approximately 1 in. $(25.4 \mathrm{~mm})$ thickness at the base. The model is made of pressed board for mass and is painted with acrylic paint to make the surface highly reflective acoustically. The model is supported on a sting that attaches to the base at the center of gravity. The sting is 48 in. (1219.2 mm) long and is wrapped with acoustic foam to minimize sound reflection, as is the mast that supports the sting. While the model is intended only to be a representation of the Blended Wing Body, its dimensions correspond approximately to $2 \%$ of the full scale size. The engine nacelle is represented by a duct made of PVC pipe of 3 in. $(76.2 \mathrm{~mm})$ inside diameter and $9.75 \mathrm{in}$. $(247.7 \mathrm{~mm})$ long. A point noise source is centered in the nacelle. The nacelle is supported separate from the wedge, so that the noise source and nacelle can be moved to different locations with respect to the fuselage.

No effort was made to simulate either the frequency spectrum or the directionality characteristics of the high bypass ratio engines that are expected to be used in the Blended Wing Body aircraft. Instead, the source used is omnidirectional and broadband in order to radiate acoustic energy over the range of frequencies that the full scale engine is expected to produce. For the inlet, tones may be expected to be generated at the blade passage frequency and its multiples. The blade passage frequency typical of a high bypass ratio engine is expected to be approximately $400 \mathrm{~Hz}$, which corresponds to $20,000 \mathrm{~Hz}$ in a $2 \%$ model, and the first harmonic of the blade passage frequency is then $40,000 \mathrm{~Hz}$. The model noise source produces measurable acoustic energy at frequencies up to $40,000 \mathrm{~Hz}$.

\section{b. Facility and Experimental Set-up}

The experiment was conducted in the Anechoic Noise Research Facility at NASA Langley Research Center. The walls of the anechoic chamber are covered with fiberglass acoustic wedges that are $3 \mathrm{ft}$. $(0.915 \mathrm{~m})$ deep and which are designed to provide $99 \%$ absorption of incident sound above $100 \mathrm{~Hz}$. The internal dimensions of the chamber (inside the tips of the acoustic wedges) are $28 \mathrm{ft}$. $(8.54 \mathrm{~m})$ by $27 \mathrm{ft} .(8.23 \mathrm{~m})$ by $24 \mathrm{ft} .(7.32 \mathrm{~m})$. A more detailed description of the ANRF is given in a NASA report by Hubbard and Manning (1983). The mounting of the model on a sting allows for the microphone array to traverse around the model so that the sound field can be measured in 3-dimensions.

A microphone traverse system is a key part of the facility, and it allows for axial and azimuthal traverses. The circular hoop that is visible in Figure 1 is mounted on a sled that can move in the 
axial direction on a linear track. The hoop contains 18 instrument-quality microphones that are equally spaced around the circumference. The microphones form a circle of 88 in $(2235.2 \mathrm{~mm})$ diameter. The hoop is rotated on $4^{\circ}$ increments so that a high resolution map of the sound field is obtained in the azimuth. The entire hoop array is positioned at 15 axial locations, spanning from 12 in. $(304.8 \mathrm{~mm})$ upstream of the leading edge of the model to $7 \mathrm{in} .(177.8 \mathrm{~mm})$ downstream of the trailing edge. The specific linear track locations are chosen such that the microphone directly beneath the model moves in $4^{\circ}$ increments with respect to the sound source in the base position. Figure 2 shows the experiment layout including the model and the microphone array.

\section{Instrumentation and Data Analysis}

The acoustic sensors mounted on the hoop array are Bruel \& Kjaer 1/4 in. (6.4 mm) diameter microphones. Noise measurements are acquired on a NEFF model 495 multi-channel data acquisition system. The data acquisition system includes Precision filters set with passband of 1,000 to $50,000 \mathrm{~Hz}$. Data are collected in blocks of 4 second duration at a rate of 100,000 samples per second. The data acquisition system is driven by a DEC Alpha computer, which is also used to save the data and for post-processing. All time histories are archived on optical disk and magnetic data tape.

All instrumentation components are calibrated on a regular schedule in accordance with ISO standards. In addition, an end-to-end calibration check of the data acquisition system is performed at the beginning and end of the test series, using a calibrated electromagnetic sound source. Samples of the time histories of each of the microphone responses are saved to files and the calibrated signals are used to check the sensitivities of the measurement microphones.

\section{d. Description of the Experiment}

The point noise source is placed in the center of the nacelle and acoustic surveys are taken around the model with the nacelle held at various locations above the model. A matrix of lateral and axial locations was evaluated by Gerhold and Clark (2001) in order to quantify the effect of nacelle location on shielding effectiveness. For the purposes of comparison to the analytic model, two axial locations, fore and aft, are discussed. In the fore location, the leading edge of the nacelle is at the axial location of the model center of gravity. In the aft location, the trailing edge of the nacelle is as far downstream of the fuselage trailing edge as it was upstream of the trailing edge in the fore position. The nacelle retains the same height when it is moved from fore to aft.

The model fuselage is then removed and acoustic surveys made with the nacelle in the same locations. In this way, the noise scattering of the model wing can be calculated directly for each of the nacelle locations.

\section{Theoretical Development}


The scattering of incident sound by an acoustically impenetrable closed surface is considered. It is assumed that linear conditions apply and all acoustic processes are time harmonic with excitation frequency $U$ and time dependence $e^{+i \square t}$. Incident sound is defined as the sound produced by a known source process in the absence of scattering surfaces. Consequently, the incident acoustic pressure field is governed by the inhomogeneous wave equation and is independent of the scattered field.

Let $B \sqcap R^{3}$ denote the closed, bounded surface of the scattering object and designate the surface interior and exterior regions by the symbols $B_{i}$ and $B_{e}$, respectively. The time independent, complex amplitude of the acoustic pressure, $P$, is split into known incident, $P_{i n c}$, and unknown scattered, $P_{s}$, components.

$$
P(\vec{x})=P_{\text {inc }}(\vec{x})+P_{s}(\vec{x}) \quad \vec{x} \square B_{e} .
$$

It is assumed that the incident acoustic pressure and its spatial derivatives are known throughout the sound field.

In the absence of flow, application of the above conditions yields a 3-D Helmholtz equation, Neumann boundary value problem for the unknown scattered pressure amplitude.
Governing PDE:
$\square^{2} P_{s}+k^{2} P_{s}=0$
$\vec{x} \square B_{e}$
Radiation Condition:$$
\lim _{r \square} \frac{\partial P_{s}}{\partial r}+i k P_{s} \mathrm{O}_{\mathrm{O}}^{\mathrm{B}}=0
$$$$
r=|\vec{x}|
$$
Boundary Condition:
$\frac{\partial P_{s}}{\partial n}=\square \frac{\partial P_{i n c}}{\partial n}$
$\vec{x} \square B$

where: $k=\frac{\square}{c}$ is the excitation wave number, $c$ the ambient sound speed, $\frac{\partial}{\partial n}$ denotes the exterior normal derivative, and $\frac{\partial P_{i n c}}{\partial n}$ is the normal derivative of the known incident pressure field.

The exterior boundary value problem is solved by employing the equivalent source method (ESM), which in essence replaces the irradiated scattering body by a system of point sources (acoustic monopoles, dipoles, etc.) strategically located in the body interior. The source strengths are adjusted so that equations ( $2 \mathrm{a}-\mathrm{c})$ are satisfied according to some rule. The ESM was chosen for its simplicity and ease of implementation, plus the fact that ESM calculations require substantially less computational resources relative to conventional numerical PDE methods. Theoretical discussion of the ESM and issues associated with its application to engineering problems can be found in Ochmann (1995), and Koopman, et. al (1989). Details of ESM implementation as they pertain to the wedge shielding experiment are given below.

\section{a. The Incident Pressure Field}

The main purpose of the shielding investigation is to determine the effect that the airframe has on scattering noise that is radiated from the engine. Thus the noise source is the engine within the 
nacelle. In the wedge scattering experiment, the engine in a nacelle is simulated by a broadband random point noise source in a cylinder that is open at both ends. To approximate the experimental source apparatus mathematically, a point monopole is placed in the middle of an infinitesimally thin, hardwall, open-ended cylinder. The theoretical configuration produces zero order circumferential duct modes that radiate from the cylinder openings at a fixed excitation frequency. The ducted fan noise prediction code TBIEM3D (Dunn (1997), Dunn, et al (1999)) is used to calculate the theoretical incident pressure field in equation (1) and the boundary condition source term in equation $(2 \mathrm{c})$.

A sample TBIEM3D calculation for the above configuration with source excitation frequency of $6300 \mathrm{~Hz}$ is presented in figure 3. Contours of instantaneous acoustic pressure and sound pressure level are plotted in a plane containing the nacelle. TBIEM3D accounts for the propagation, reflection at the duct openings, and radiation to the farfield of the monopolegenerated sound.

\section{b. The Scattered Pressure Field}

Several variations of the ESM occur in practice and are surveyed by Ochmann (1995). In the current application, a collection of point monopoles is used to simulate the scattered acoustic pressure field. The sources are distributed on an auxiliary surface located inside the wedge and the source strengths adjusted so as to minimize a certain boundary condition error function on the actual wedge surface. The auxiliary source surface is an $85 \%$ scaled replica of the wedge that is concentric with the wedge. The actual and source surfaces are discretized by triangulating the wedge faces as illustrated in figure 4. Point monopoles are situated at the $M$ vertices of the source surface and the acoustic boundary condition is evaluated at the $N$ actual wedge surface vertices $(M<N)$. This approach was chosen for simplicity and minimal computer usage. Adding higher order terms (dipoles, quadrupoles, etc.) to the equivalent source collection improves ESM accuracy, but increases the computational complexity and was not considered in this research.

The acoustic pressure field produced by a point monopole with unit strength at the location $\vec{x}_{j}$ is given by the formula

$$
P_{j}(\vec{x})=\frac{1}{4 \square} \frac{e^{\square i k R_{j}}}{R_{j}},
$$

where

$$
R_{j}=\left|\vec{x} \square \vec{x}_{j}\right| .
$$

Note that (3) is a solution of (2a-b). An approximation to the scattered pressure field is constructed by forming a superposition of the fields produced by the monopole distribution. Let the sequence $\left\{\vec{x}_{j}\right\}_{j=1}^{M} \square B_{i}$ denote the vertices of the triangulated source surface, then

$$
P_{s}(\vec{x}) \square \bar{P}_{s}(\vec{x})=\bigsqcup_{j=1}^{M} a_{j} P_{j}(\vec{x}) .
$$


The approximate solution, $\bar{P}_{s}$, satisfies (2a-b), and the unknown source strengths, $a_{j}$, are determined by applying the boundary condition (2c). A fundamental advantage of this approximation is that once the coefficients are calculated, (5) is easily evaluated anywhere in the sound field.

Define the boundary error function $\measuredangle(\vec{x})$ by the formula

$$
\square(\vec{x})=\frac{\partial \bar{P}_{s}}{\partial n}(\vec{x})+\frac{\partial P_{i n c}}{\partial n}(\vec{x}) \quad \vec{x} \square B .
$$

The error function is minimized by adjusting the monopole source strengths according to some prescribed rule. Several methods for minimizing the boundary error appear in practice and have been assessed by Ochmann (1995). These techniques vary in numerical complexity, accuracy, and physical significance. In this research, the error function is set equal to zero at the $N$ vertices of the triangulated actual surface. This approach was adopted due to the need for computational expediency.

Let $\left\{\vec{y}_{i}\right\}_{i=1}^{N} \square B$ represent the Cartesian coordinates of the discretized actual surface vertices, then applying equation (5) to the error function at these points produces the overdetermined system of linear equations

$$
\square_{j=1}^{M} a_{j} \frac{\partial P_{j}}{\partial n}\left(\vec{y}_{i}\right)=\square \frac{\partial P_{i n c}}{\partial n}\left(\vec{y}_{i}\right) \quad i=1, \ldots, N
$$

for the unknown coefficients, which is solved using linear least squares techniques.

\section{c. ESM Validation}

The validity and noise prediction capabilities of the ESM+TBIEM3D noise prediction system are demonstrated by evaluating the calculated acoustic pressure field on a cylindrical surface that encloses the wedge. The dimensions and positioning of the cylindrical field surface simulates the placement of the experimental microphone hoop and facilitates comparison with experimental results. All calculations were performed on a PC with a $350 \mathrm{MHz}$ Pentium II processor and 256 MB RAM

A sample shielding calculation using the ESM is shown in figure 5. In this calculation, the nacelle has been removed and a point monopole radiates incident sound at $3000 \mathrm{~Hz}$. The point source is located over the aft triangular surface aft of the peak of the wedge. Figure 5 shows the instantaneous acoustic pressure field plotted on the cylindrical envelope and viewed from three orientations. In the non-oblique views, the pressure contours are made transparent so that the wedge outline is visible. Reflection, diffraction, and shielding of the incident sound by the wedge surfaces and edges are evident. In particular, the upward reflection of sound by the aft triangular surface and the appearance of a shadow region beneath the wedge are clearly depicted.

\section{Results}


Sound level surveys were taken with the nacelle alone and with the nacelle in the presence of the wedge. The shielding effect of the wedge shape is defined as the difference between measured sound pressure level (SPL) with the nacelle alone and with the wedge in place. The shielding is calculated analytically in a similar manner. The incident SPL map is calculated using TBIEM3D and the scattered SPL map is calculated with the wedge present using the ESM. Then noise shielding is evaluated from the difference of these two contour maps. Narrow-band FFTs $(\square \mathrm{f}=$ $6.1 \mathrm{~Hz}$ ) are performed on the measured acoustic data and are presented in this paper at $6300 \mathrm{~Hz}$ and $8000 \mathrm{~Hz}$ for comparison with the analytical results. The upper frequency limitation is imposed by the capacity of the computer on which the analysis was run. These frequencies correspond to $126 \mathrm{~Hz}$ and $160 \mathrm{~Hz}$, respectively in the full scale. Although this is a relatively low frequency band, it is felt that the results are sufficient to compare the theory and experiment. The broadband ( $1 \mathrm{kHz}$ to $25 \mathrm{kHz}$ in the model scale) overall sound pressure level (OASPL) is also evaluated from the experimental data. The OASPL corresponds to the broadband noise of the spectrum without the influence of tones and generally accentuates the influence of low frequency noise sources. An additional experimental sound survey is performed with the point noise source alone in the center of the hoop array. This survey is intended to measure the directivity of the source alone. Gerhold and Clark (2001) present experimental results from the full matrix of engine nacelle locations and at a higher frequency, which corresponds to the blade passage frequency of the ducted fan that would be expected to power the Blended Wing Body.

\section{a. Point Noise Source}

The noise source is comprised of four impinging air jets, shown in Figure 6 without the nacelle. The impinging jet arrangement is designed to provide a broadband high frequency, high intensity, omnidirectional noise source that is well suited to scale model work. A representative spectrum of the noise emitted by the impinging jet source is shown in the upper curve in Figure 7 . The source is relatively broadband from frequencies below $6000 \mathrm{~Hz}$ to 40,000 Hz. The lower curve in Figure 7 is the background ambient sound level in the anechoic chamber. It is seen that, at frequencies from $10 \mathrm{kHz}$ on out, the impinging jet source produces noise that is at least $10 \mathrm{~dB}$ above the background ambient. Figure 8 shows a map of the directivity of the OASPL of the point source measured in the anechoic chamber. The directivity plot shows a four lobe pattern which comes from the four jets. The scale has been exaggerated to show the details of this lobe pattern, and the figure shows that the variation in sound level at any azimuth is no more than 2.0 $\mathrm{dB}$.

\section{b. Comparison of Experimental and Analytical Results}

Figure 9 shows the noise radiation from the point source in the nacelle, without the wedge in place, at $6300 \mathrm{~Hz}$. The map on the left is measured and the map on the right is calculated. The outline of the nacelle is shown in the figure. The patterns of radiation are similar between experiment and analysis, in particular compare the lobed patterns of the sound radiation for the inlet and exhaust ends of the nacelle. The axial separation of the sound lobes is not as clear in the 
experiment as it is in the analytic results. This is due to the limited axial resolution that arises from the number of axial stations at which data were taken.

Figure 10 is the estimated sound pressure level map with the wedge in place. The outline of the wedge and nacelle are shown in the figure in order to show the orientation of the nacelle with respect to the wedge. The orientation in this instance is designated as nacelle in the center location laterally and forward location axially. The similarity between footprint of noise reduction beneath the model for the experiment and analysis is evident. The increased noise radiation behind and below the model is also similar.

Figure 11 is the comparison of noise scattering by the wedge airframe at $6300 \mathrm{~Hz}$. The noise is reduced by approximately $10 \mathrm{~dB}$ in the shadow zone directly below and upstream of the wedge shape. The outline of this shadow zone is shown more clearly from the analytic results, but is evident in the experimental results as well. The noise is reduced in the area directly downstream of the trailing edge, although it is less than the noise reduction upstream. Further downstream and directly behind the engine, the ability of the airfoil to shield noise radiation is negligible. However, off the sides, around $90^{\circ}$ azimuth, the noise is actually increased. This arises from the reflection of aft-radiated sound by the sloping rear surface of the airframe. It is indicated in both the experimental and analytic contours. A lobe of sound radiation is seen below the model and upstream from the trailing edge. This lobe is seen in the experimental and the analytic results.

Figure 12 shows the noise radiation from the point source in the nacelle, without the wedge airfoil in place, at $8000 \mathrm{~Hz}$. The analytic radiation pattern shows a clear pattern of lobes. The axial distance separating the maxima on the nacelle centerline is approximately 5.5 wavelengths (at $8000 \mathrm{~Hz}$ ). This compares to a 5.9 wavelength (at $6300 \mathrm{~Hz}$ ) separation of the maxima at the 6300 $\mathrm{Hz}$ radiation case (figure 9). The experimental results lack the axial resolution to distinguish the lobed pattern.

Figure 13 is the comparison of noise scattering by the wedge airframe at $8000 \mathrm{~Hz}$. The noise is reduced by approximately $10 \mathrm{~dB}$ in the shadow zone directly below and upstream of the wedge shape. The outline of this shadow zone is comparable between the analytic and experimental results. The gradual reduction of shielding downstream of the trailing edge of the airfoil is seen in both contours. The scattering of sound into the area further behind the airfoil that is clearly shown in the analytic results is not seen in the experimental results. The scattering of sound into the region above the model and upstream of the nacelle is clearly seen in both contours.

One of the purposes of this experiment is to investigate the effect of nacelle location on the shielding efficiency of the BWB airfoil. Figure 14 shows the noise scattering at $6300 \mathrm{~Hz}$ by the wedge-shaped airfoil for the engine nacelle in the center-aft location. When the nacelle is in the aft location, the discharge end extends beyond the trailing edge of the wing. The azimuthal extent over which noise is reduced in the forward quadrant below the wedge is wider than it was with the nacelle in the forward position, but the noise reduction directly below the trailing edge is less. There is an area slightly downstream of the trailing edge, beneath as well as above the model, in 
which the noise is amplified. The experimental and analytic results are comparable and exhibit similar sensitivity to the effects of source location.

\section{Conclusions}

The main purpose for this research effort is to develop the noise scattering analytical model and to validate it experimentally. The model combines the three-dimensional Boundary Integral Equation Method (TBIEM3D), which estimates the incident sound from the engine noise source, with the Equivalent Source Method (ESM), which determines the scattering by the reflecting body to synthesize the sound field in the vicinity of the airfoil. An experiment is developed in parallel with the analytic program, the purpose of which is to provide measured noise scattering data with which to validate the analytic model. The airframe is a wedge-shape comprised of planar surfaces. The purpose is to provide the major features of the Blended Wing Body concept with the simplest design, for implementation into the analysis. The engine is represented by a broadband, high frequency point source in a nacelle. The nacelle is mounted independent of the airframe so that the effect of engine location can be investigated.

The results of both the experiment and the computation show that scattering and diffraction of sound are found to be sensitive to location of the source and to the shape of the airframe. Maximum noise reduction is found to occur in the forward sector beneath the airfoil. The tapered sides of the upper surface of the airfoil scatter incident sound forward and above the model when the source is located in the forward position, and toward the rear when the source is in the aft position. The experiment and computation agree, not only in the overall shielding characteristics but also in some finer structural details of noise scattering. Spatial resolution in the analytical model is finer than it is in the experiment. In addition, the computations are at a discrete frequency whereas the experimental results cover a narrow but finite frequency band. Because of these resolution differences, the estimated noise radiation shows the presence of sound wave patterns more clearly than does the measurement. If the assessment of impact of more subtle configuration changes than those considered here becomes an issue, it will be necessary to increase the resolution of the measurements. The computational results were limited to relatively low frequencies because of constraints imposed by the computer on which the analysis was performed. It will be necessary to improve the computing capability in any future analyses in order that the computational results can be more useful in terms of assessing the impact on ground-based observers of noise shielding by the Blended Wing Body.

\section{Acknowledgements}

The authors are grateful for contributions made to the successful completion of this project by Beverly Anderson of NASA LaRC and Phil Grauberger of Wyle Laboratories for experiment set up and data collection, and by Lawrence Becker, Florence Hutcheson, and Jeffrey Kelly of Lockheed-Martin for data reduction. 


\section{References}

Clark, L.R. and Gerhold, C.H., "Inlet Noise Reduction by Shielding for the Blended-Wing-Body Airplane," AIAA Paper no. 99-1937, presented at the $5^{\text {th }}$ AIAA/CEAS Aeroacoustics Conference, May 10-12, 1999, Seattle, WA.

Dunn, M.H.: TBIEM3D - A Computer Program for Predicting Ducted Fan Engine Noise, Version 1.1. NASA/CR-97-206232 [1997].

Dunn, M.H., Tweed, J., Farassat, F., “The Application of a Boundary Integral Equation Method to the Prediction of Ducted Fan Engine Noise," Journal of Sound and Vibration, vol. 227, number 5 [1999], pgs. 1019-1048.

Gerhold, C.H. and Clark, L.R., 'Database of Inlet and Exhaust Noise Shielding for a WedgeShaped Airframe," NASA/TM-2001-210840, April, 2001.

Hubbard, H.H. and Manning, J.C., "Aeroacoustic Research Facilities at NASA Langley Research Center,” NASA TM 84585, March, 1983.

Koopmann, G., Song, L., and Fahnline, J.: "A Method for Computing Acoustic Fields Based on the Principal of Wave Superposition," Journal of the Acoustical Society of America, vol 86 [1989]. pgs. 2433-2438.

Ochmann, M.: “The Source Simulation Technique for Acoustic Radiation Problems," Acustica, vol. 81 [1995], pgs. 512-527. 


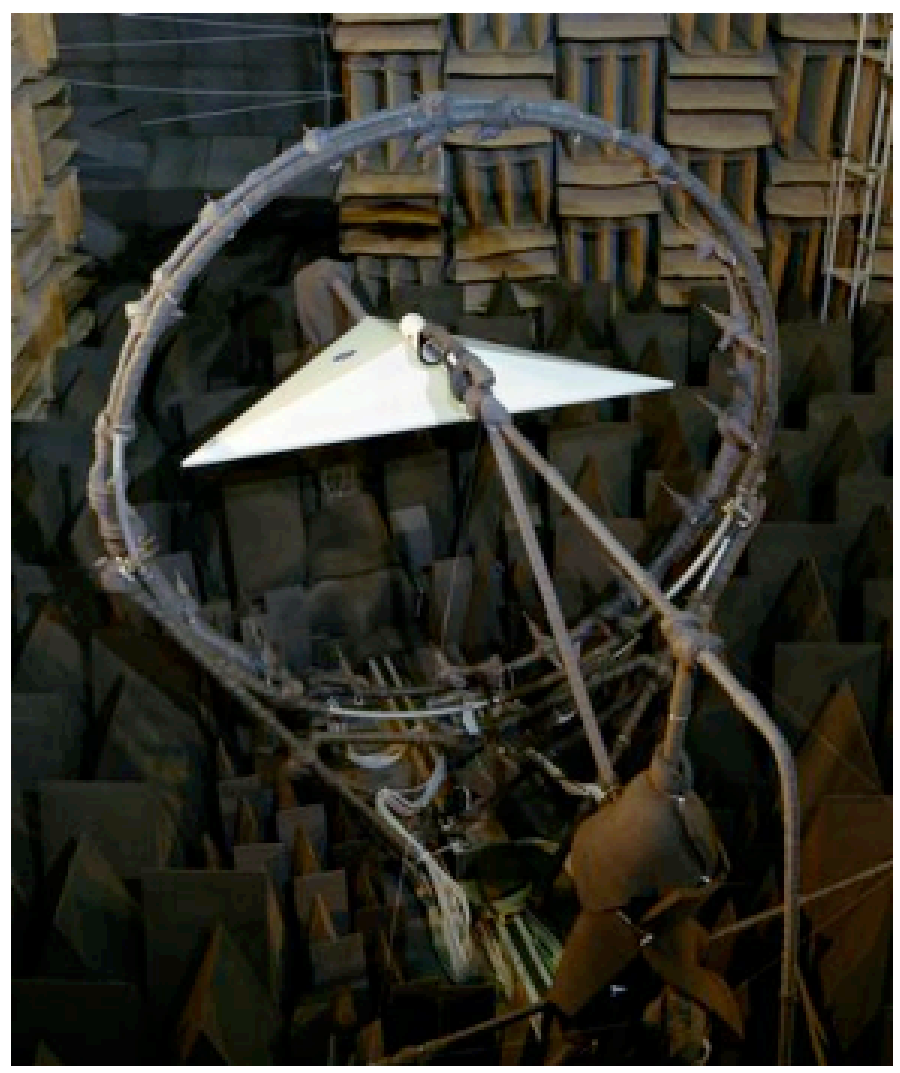

Figure 1. Photograph of wedge-shape airfoil model experiment set-up in anechoic chamber, view looking upstream
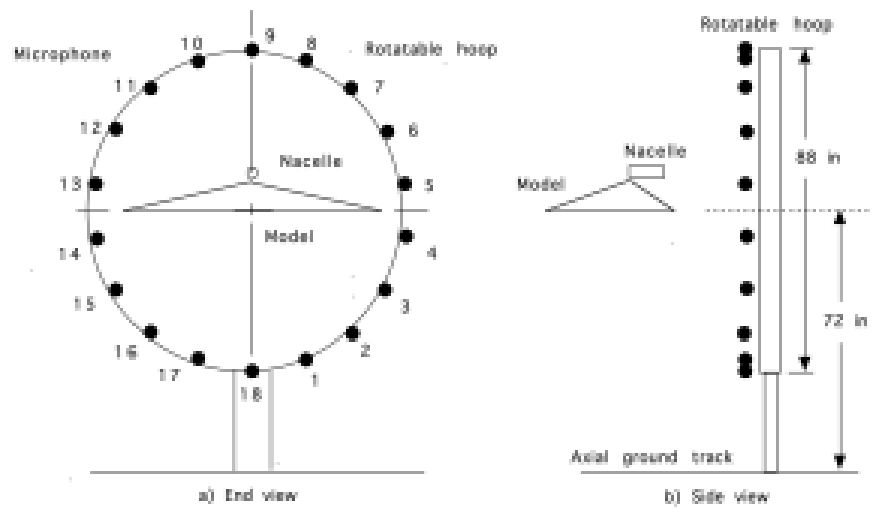

Figure 2. Sketch of exp eriment set-up showing traversing microphone array and wedge-shaped airfoil. 


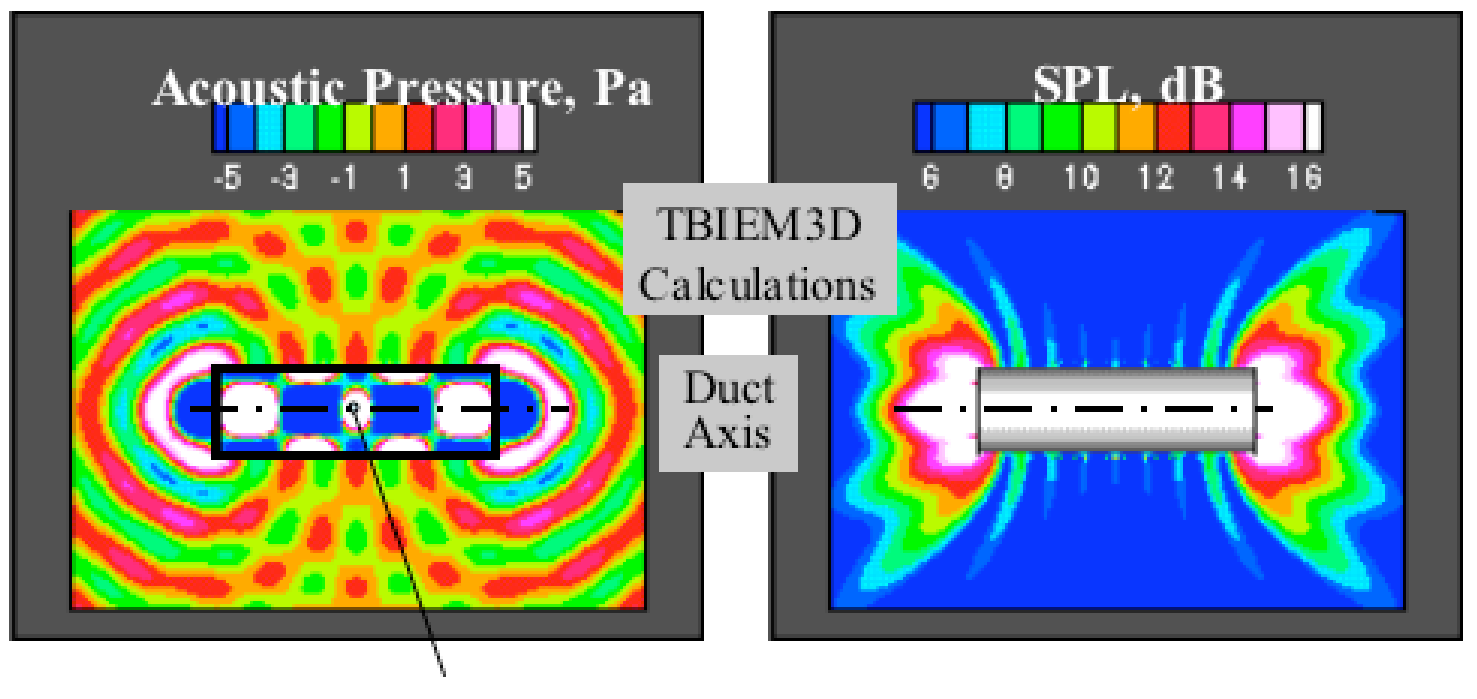

Point Monopole $-\mathrm{f}=6300 \mathrm{~Hz}$

Figure 3: Acoustic Near-Field for a Point Monopole Source Inside a Cylinder (a) Acoustic Pressure Contours - Duct Cut-Away View

(b) Sound Pressure Level Contours

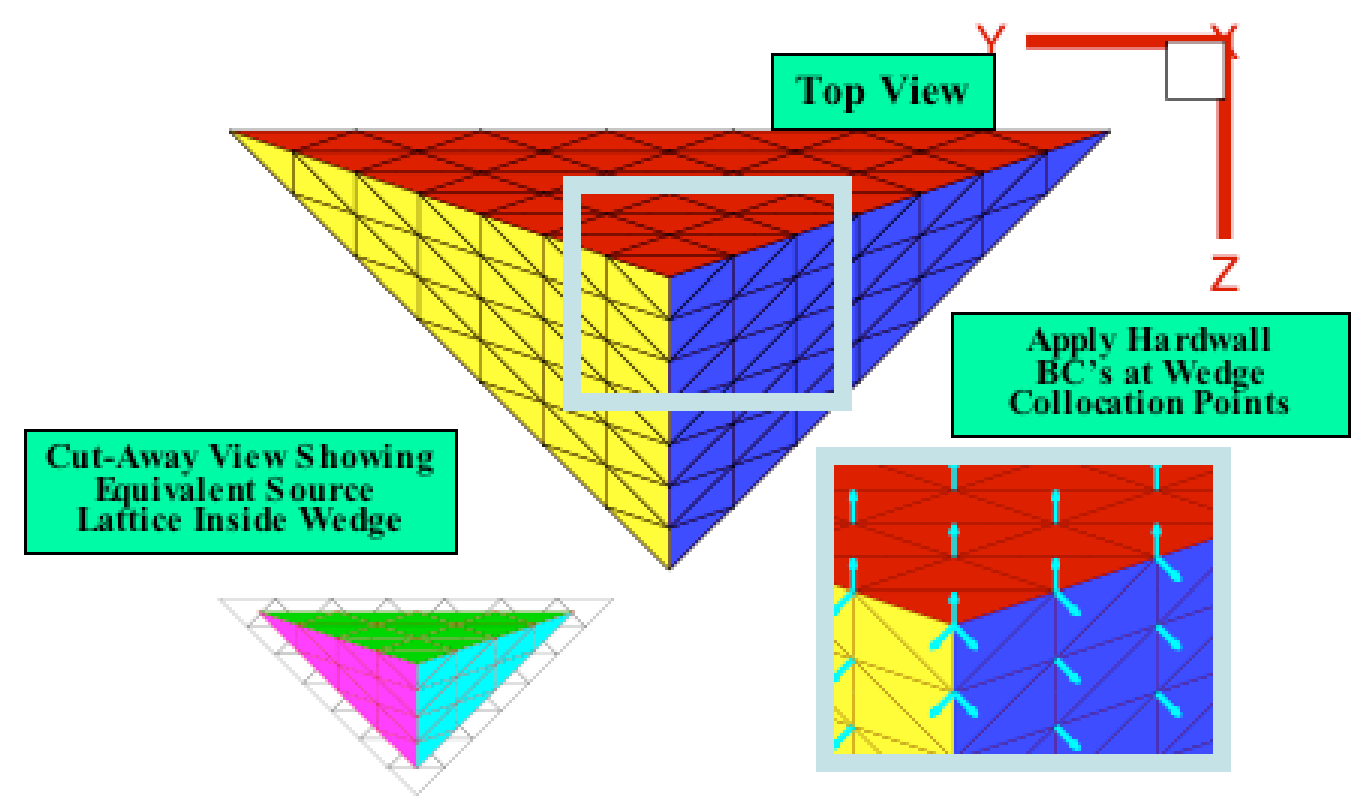

Figure 4: Wedge Discretization for ESM Calculations (Coarse Grid) 


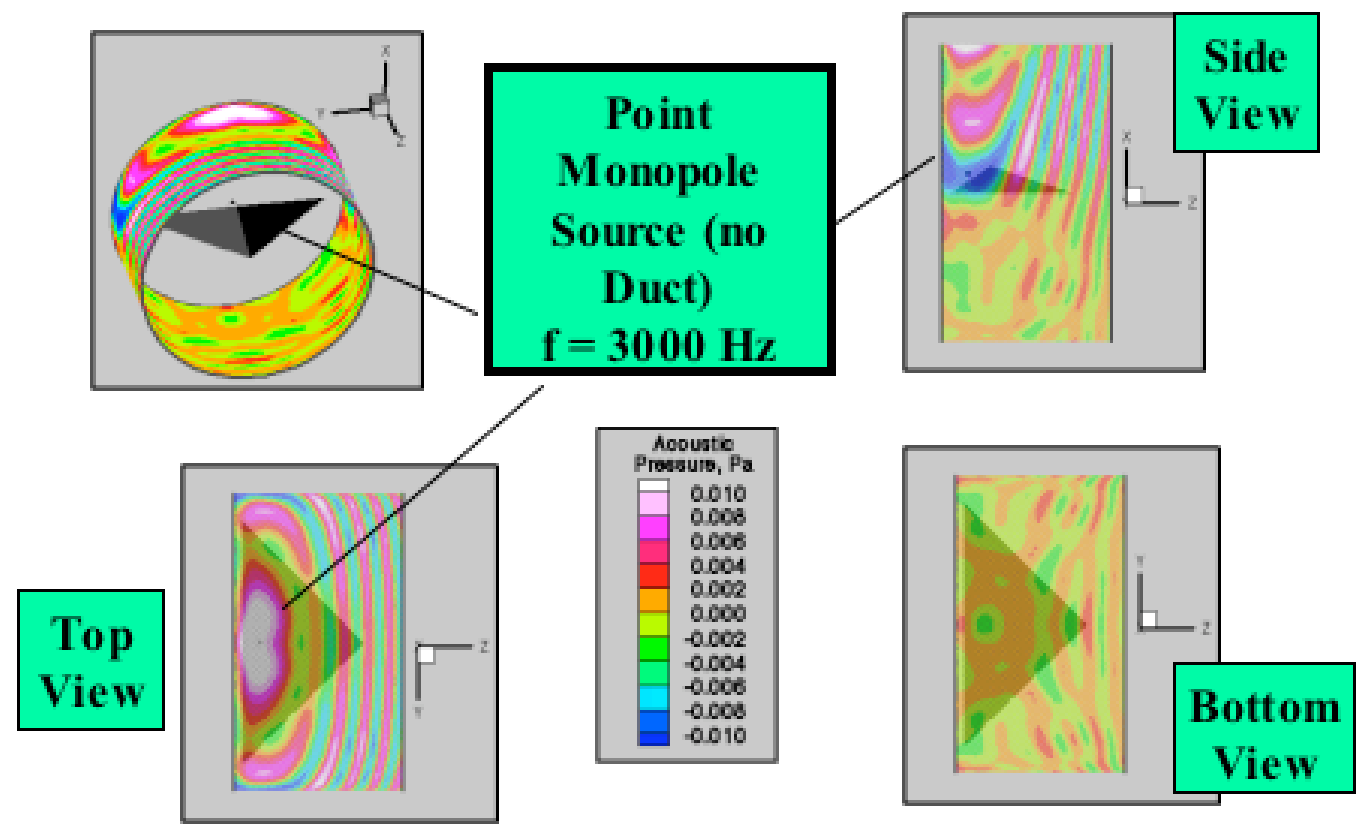

Figure 5: Sample ESM Calculation for Scattering of Incident Sound by a Wedge Acoustic Pressure Contours Plotted on Cylindrical Surface

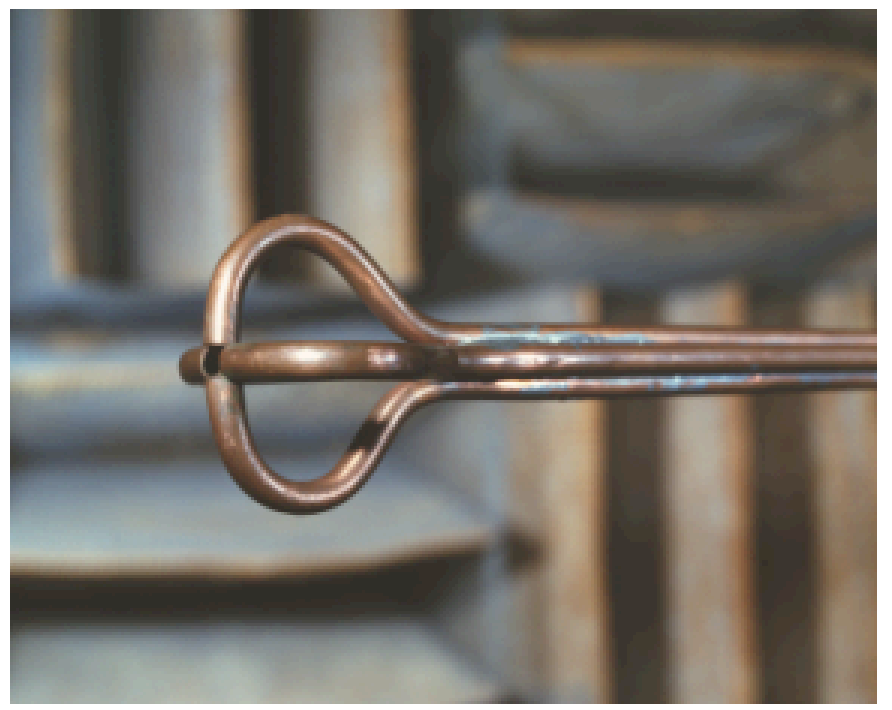

Figure 6. Broadband, high intensity point noise source consisting of four impinging air jets. 


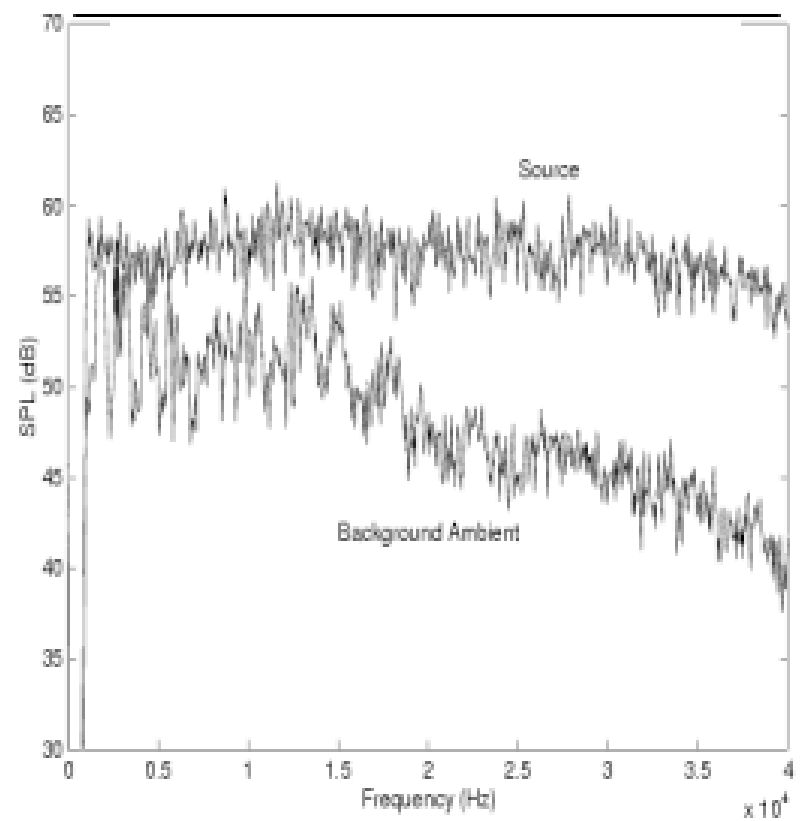

Figure 7. Narrowband sound level spectrum for imp inging jet point source

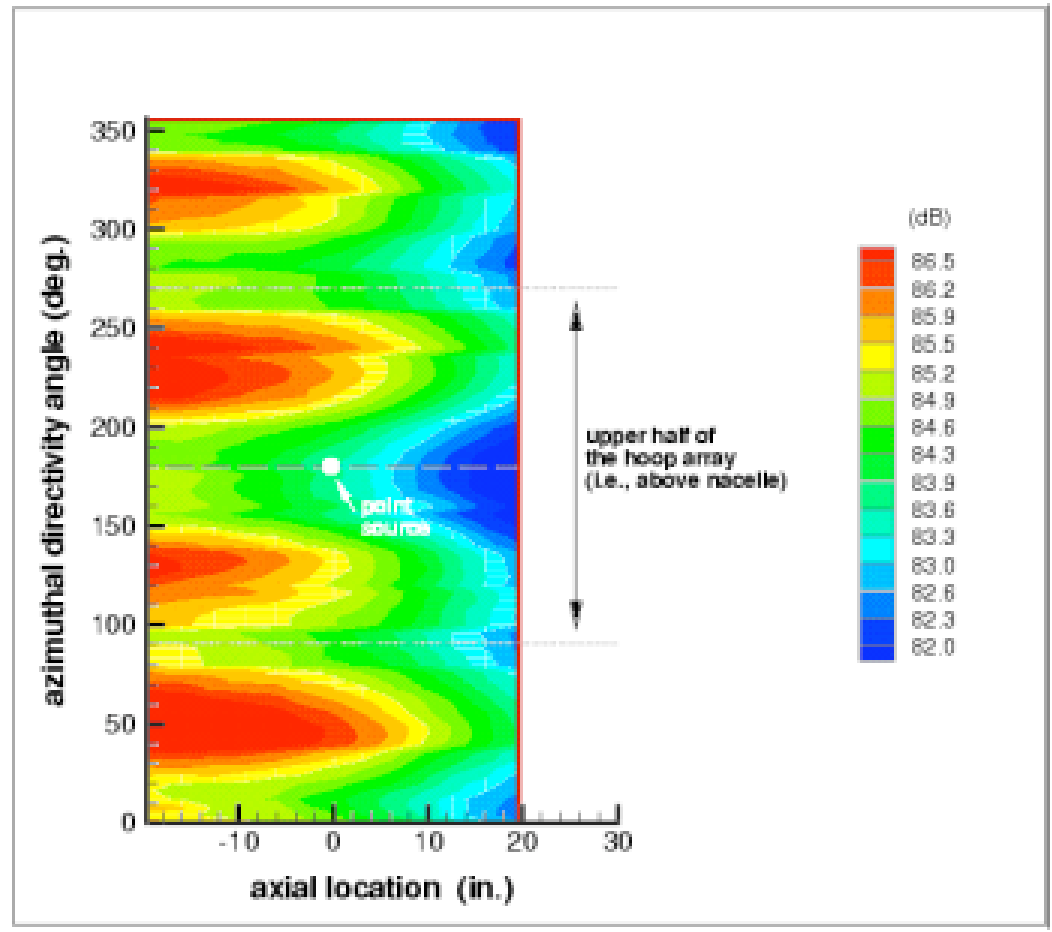

Figure 8. OASPL directivity contour of impinging-jet point noise source. 


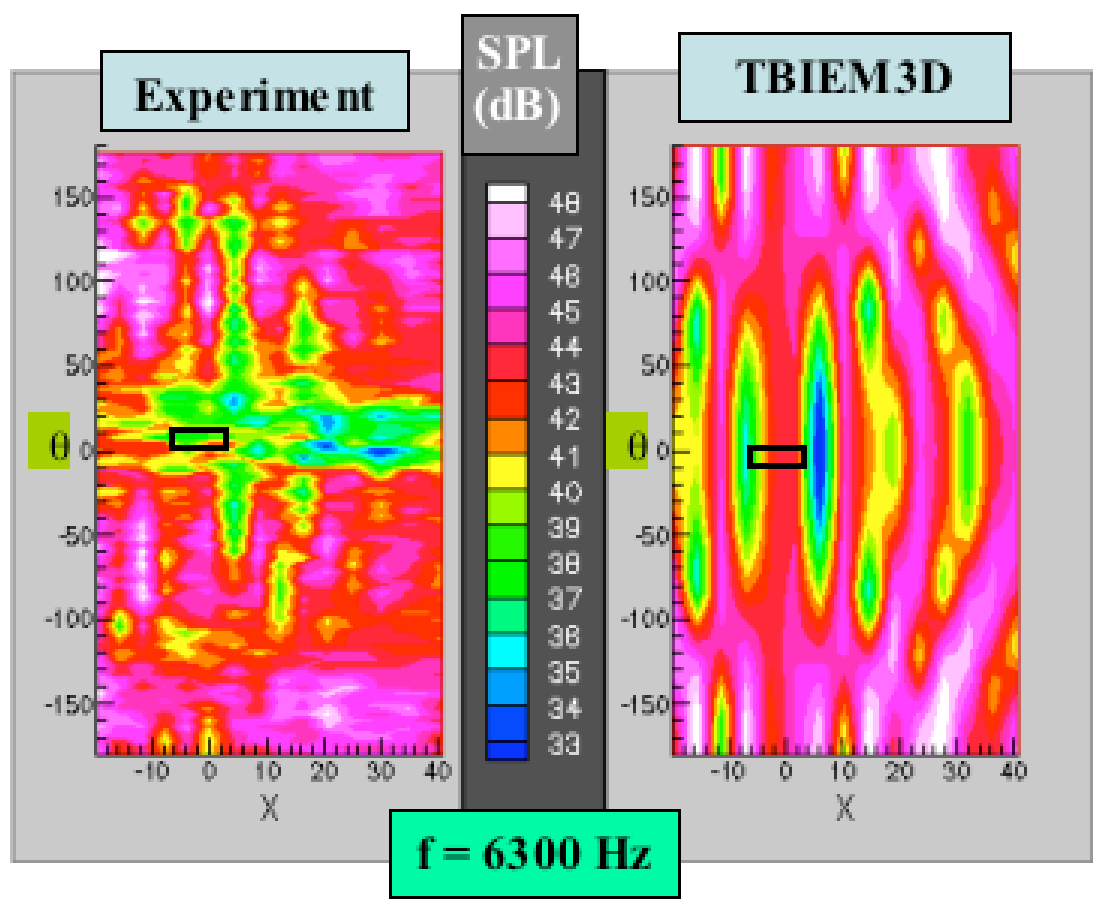

Figure 9: Comparison of experimental and analytic contours of point noise source sound radiated from inlet and discharge ends of a nacelle at $6300 \mathrm{~Hz}$.

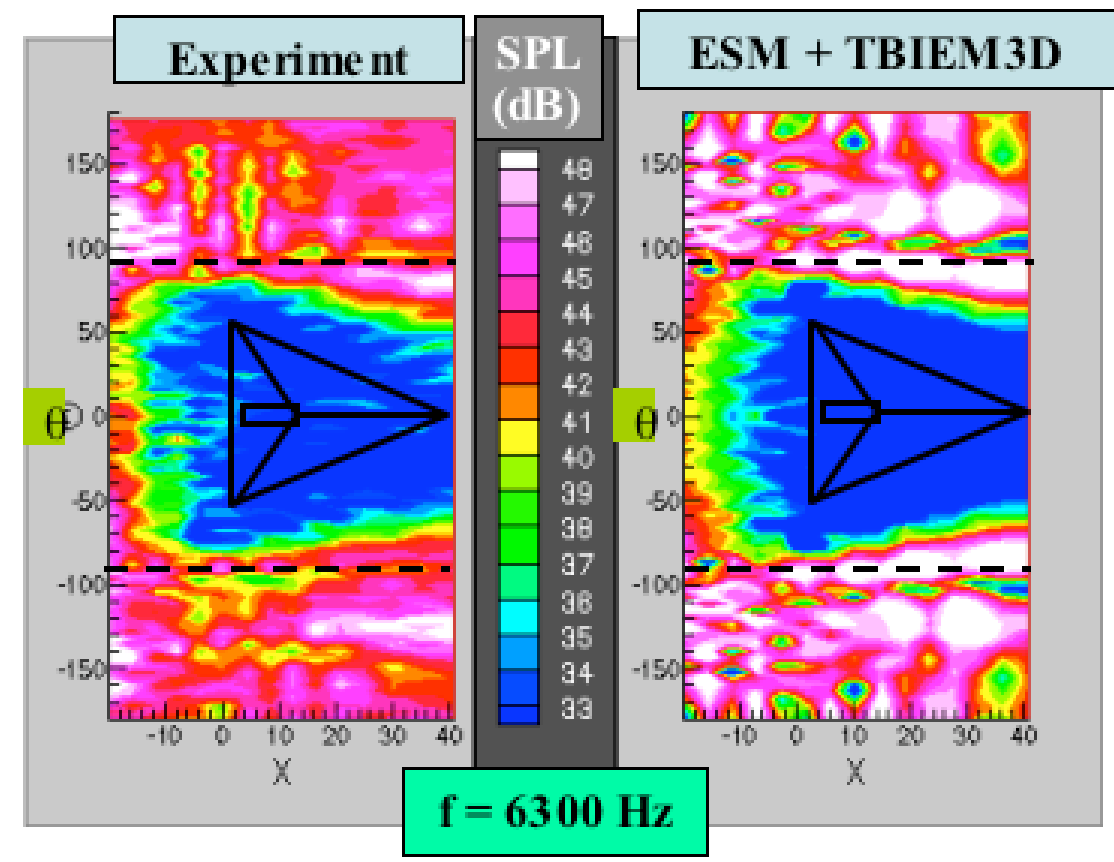

Figure 10: Comparis on of experimental and analytic contours of nacelle-radiated sound in the vicinity of the wedge-shaped airfoil at $6300 \mathrm{~Hz}$, nacelle in center-forward position 


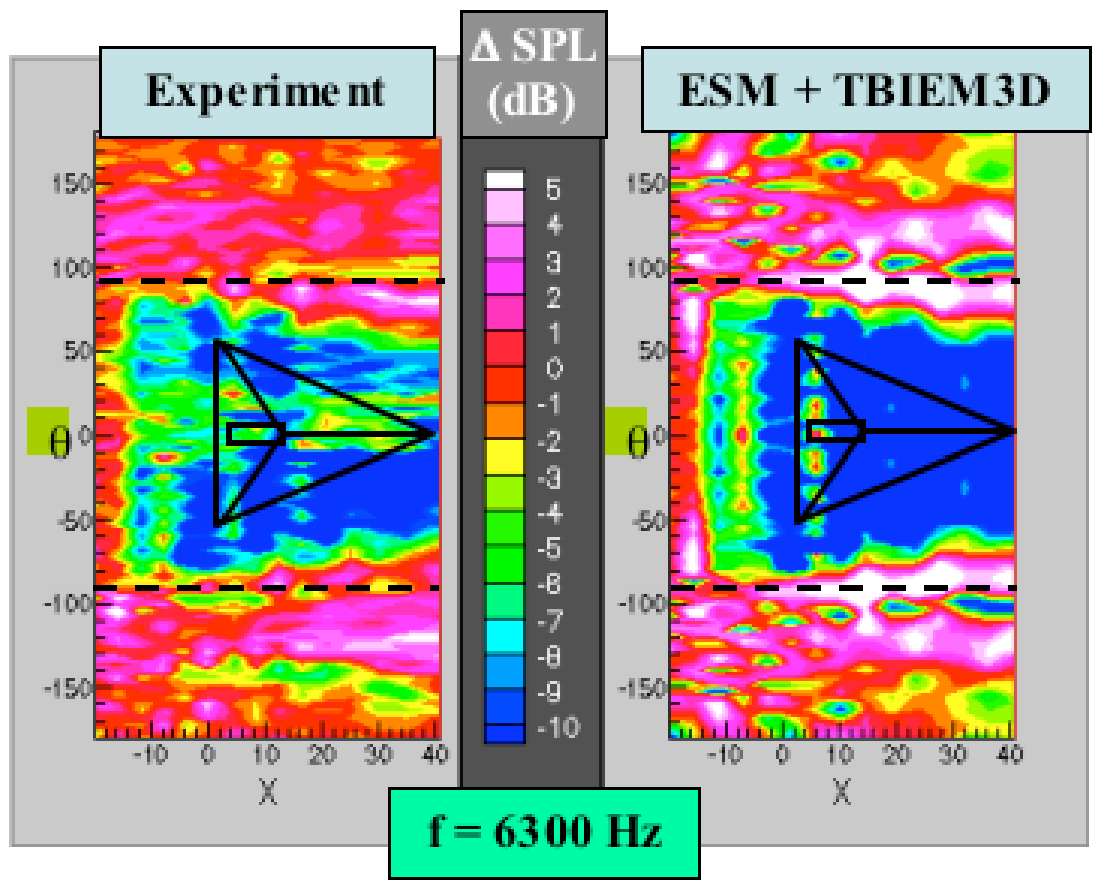

Figure 11: Comparison of experimental and analytic contours of sound scattering effect of a wedge-shaped airfoil at $6300 \mathrm{~Hz}$, nacelle in center-forward position

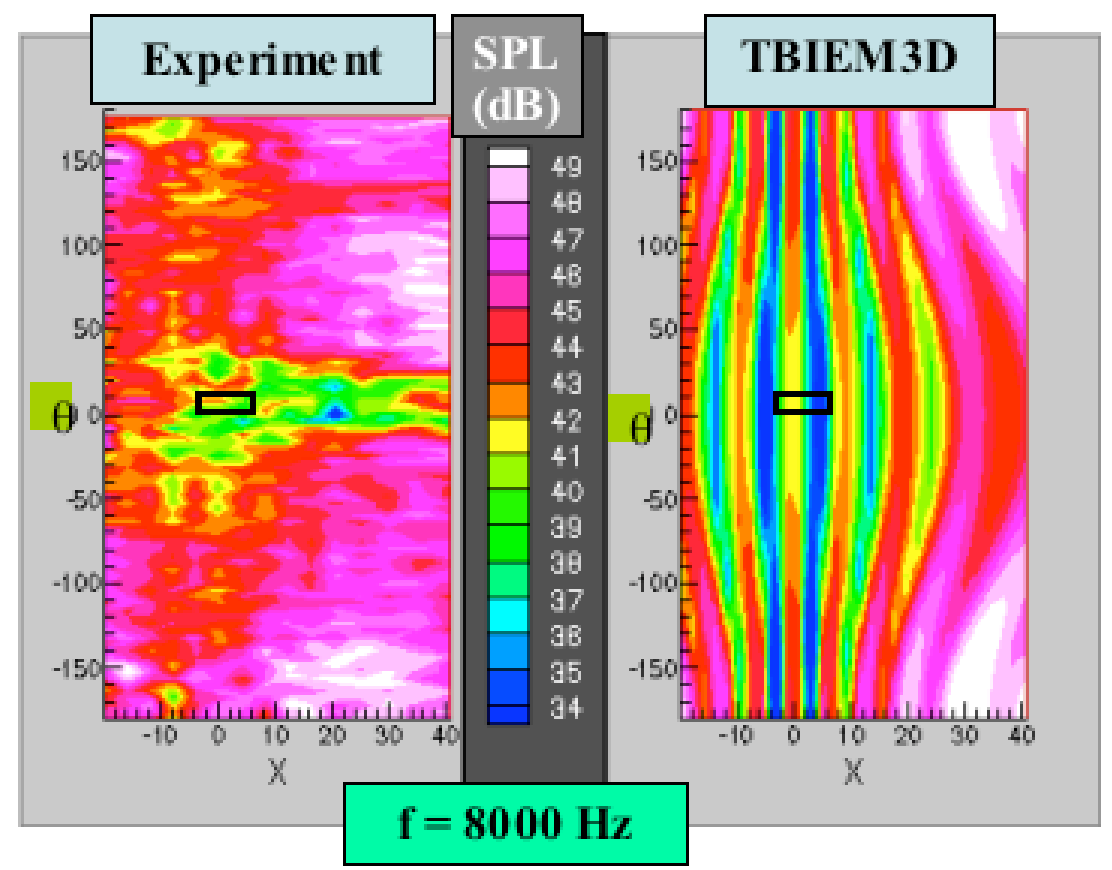

Figure 12: Comparison of experimental and analytic contours of point noise source sound radiated from inlet and discharge ends of a nacelle at $8000 \mathrm{~Hz}$. 


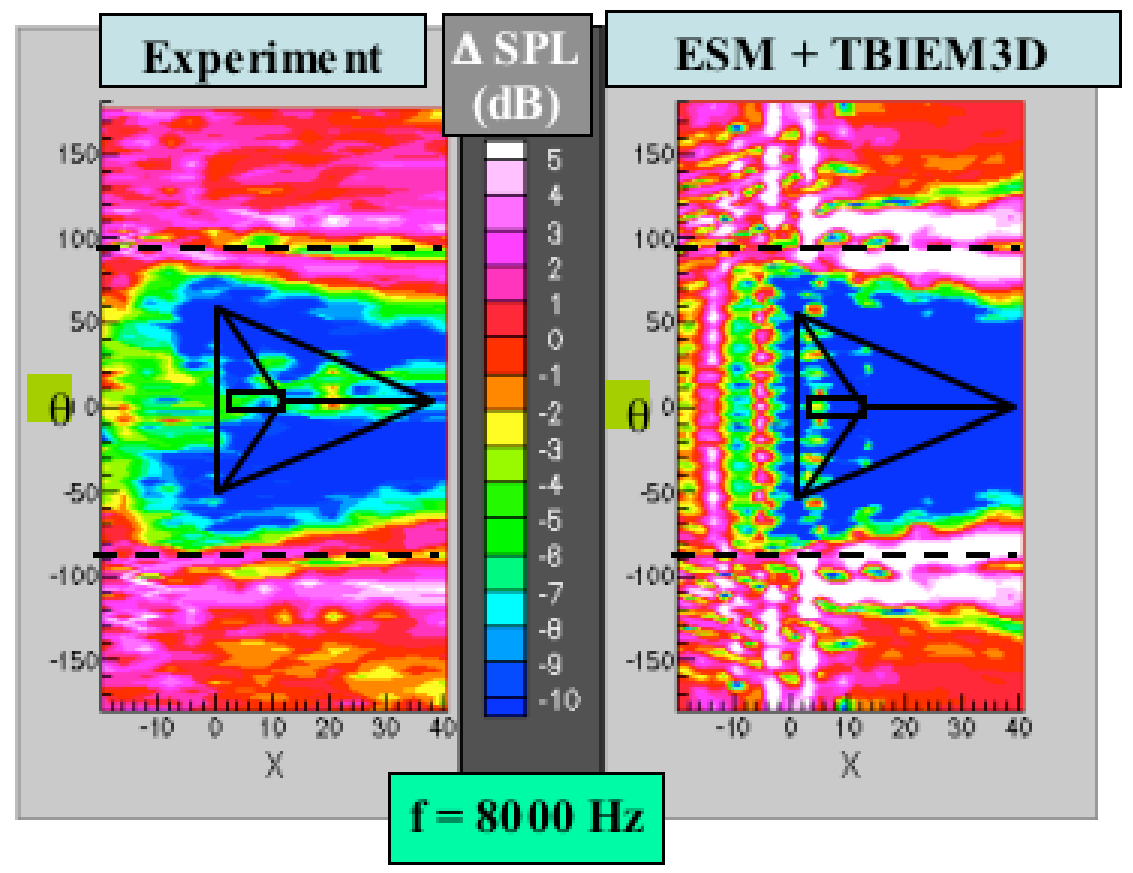

Figure 13: Comparison of experimental and analy tic contours of sound scattered by wedge-shaped airfoil at $8000 \mathrm{~Hz}$, nacelle in center-forward position

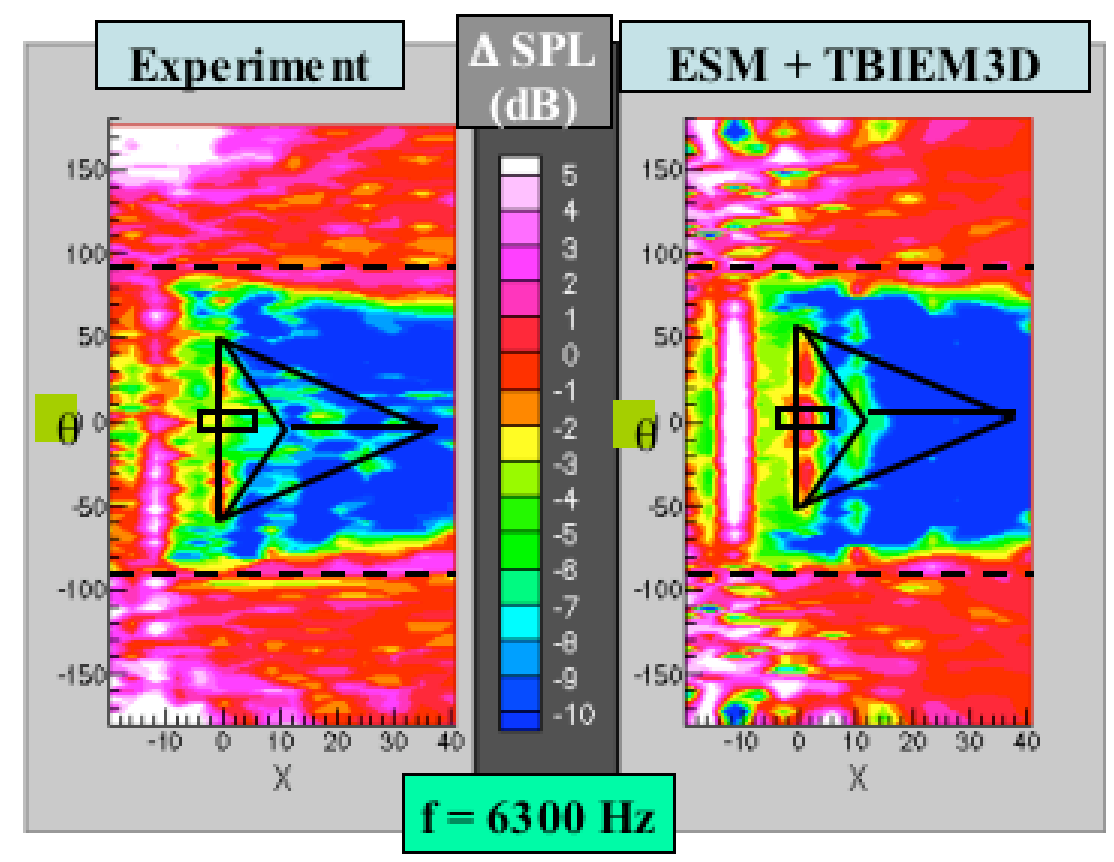

Figure 14: Comparison of experimental and analytic contours of sound scattered by wedge-shaped airfoil at $6300 \mathrm{~Hz}$, nacelle in center-aft position 